\title{
Experimental Approach to Explosive Hydrogen Burning in X-Ray Bursts and Core-Collapse Supernovae
}

\author{
Shigeru Kubono ${ }^{1-3^{*}}$ \\ ${ }^{1}$ RIKEN Nishina Center, 2-1 Hirosawa, Wako, Saitama, 351-0198 Japan \\ ${ }^{2}$ Institute of Modern Physics, Chinese Academy of Sciences, Lanzhou, China \\ ${ }^{3}$ Center for Nuclear Study, the University of Tokyo, 2-1 Hirosawa, Wako, Saitama, 351-0198 Japan
}

\begin{abstract}
Recent experimental challenges to study the explosive hydrogen burning at extremely high temperatures are discussed. Along the nucleosynthetic pathway, discussions were made especially for the medium mass region, where we have possible waiting points and bottle necks which influence not only the light curve but also the production of heavy elements including the anomalously abundant p-nuclei at $\mathrm{A}=80$ 100. Two approaches were discussed for the problem; one is the precision mass measurements and the other one is the half-live measurement of very short lived isotopes. A scope of the field is also discussed.
\end{abstract}

\section{Explosive Hydrogen Burning at Extremely High Temperatures}

Since hydrogen is the most abundant element, there are some sites where hydrogen burns explosively [1]. Typical such nucleosyntheses include the rapid proton-capture process (rp-process) in type I X-ray bursts, and the vp-process in type II supernovae. Type I $\mathrm{X}$-ray burst will take place typically in a binary system involving a neutron star $[2,3]$.

The property of neutron stars seems quite interesting, but is poorly known. Binary system of stars provide a good occasion to study the property of neutron stars. One is the type I X-ray burst, and other is the neutron star merger which was recently observed in August 2017, providing observations of the gravitational wave[4] and the following kilo nova phenomena [5]. The type I X-ray burst will take place by thermonuclear run away with accreted gas from the accompanying star onto the surface of the neutron star. It also provides an occasion to learn possibly the elemental evolution in the crust region of the neutron stars.

The second nucleosynthetic scenario, the vp-process, proposed in 2006 by three groups [6-8], is considered to take place in the early epoch of type II supernovae, where the site can be slightly proton-rich because of the mass difference of proton and neutron. This process is considered to produce very efficiently neutron-deficient nuclei in the light and medium mass regions. Thus, it is expected to make a significant contribution to the chemical evolution.

\footnotetext{
* Corresponding author: kubono@riken.jp
} 
Therefore, this process is of great interest especially for production of the p-nuclei in the intermediate mass region $(\mathrm{A}=80 \sim 100)$ [9], which are anomalously abundant isotopically. However, the nucleosynthetic flow path and the nuclide productions are largely uncertain because nuclear physics of the pathway region on the nuclear chart are still not known well yet $[10,11]$. Extensive mass measurements have been reported in the past decade, but not completed yet. Some examples are found in refs. $[12,13]$.

Both the rp-process and the vp-process run through similar mass region in the neutrondeficient nuclear region, and have the common problems. However, the rp-process would have waiting points and bottle necks along the pathway, whereas the $v p$-process may have less effects at the points due to the neutron-induced reactions, $(n, p)$ and $(n, \gamma)$, which bypass the long-waiting beta-decays [10]. The waiting points also have a chance to be bypassed by the two-proton capture process (2p-process) [13] in both scenarios. Thus, the experimental challenges have been made in the past years to determine the rough pathway by precision mass measurements.

In the present lecture, I discuss recent experimental efforts around the possible waiting points in the rp-process, and production of the p-nuclei in the vp-process in the medium mass region.

\section{Waiting Points at Intermediate Mass Region}

There are many works reported for the nucleosynthesis at $\mathrm{A}<56$ in the rp-process, including experimental works using stable and radio-active nuclear beams [1]. Figure 1 illustrates the possible nucleosynthesis flow beyond ${ }^{56} \mathrm{Ni}$, indicating possible waiting points and the bottle-necks. Sensitivity tests of each reaction along the rp-process pathway were also reported [14], suggesting crucial roles of nuclear reactions around the waiting points for heavy element synthesis, as shown in Fig. 1. The red squares in Fig. 1 are the possible waiting point nuclei, although none of them are well understood yet.

In this mass region, the proton capture reaction, $\mathrm{A}(\mathrm{p}, \gamma) \mathrm{B}$, and the beta decay are the main processes for the rp-process. The reaction of $A(p, \gamma)$ has a certain flow-back depending on the proton separation energy in nucleus $\mathrm{B}$, i.e., whose relative contribution is defined by the Boltzmann factor, $\exp (-\mathrm{Q} / k \mathrm{~T})$, where $\mathrm{Q}$ is the proton separation energy, $k$ is the Boltzmann constant, and $\mathrm{T}$ is the temperature of the site in kelvin [15]. At higher temperature with high density, the $2 \mathrm{p}$-process may proceed to make a nucleus $\mathrm{A}+2 \mathrm{p}$. For

Fig. 1. The rpprocess flow predicted, where $t$ red squires indical possible waiting points.

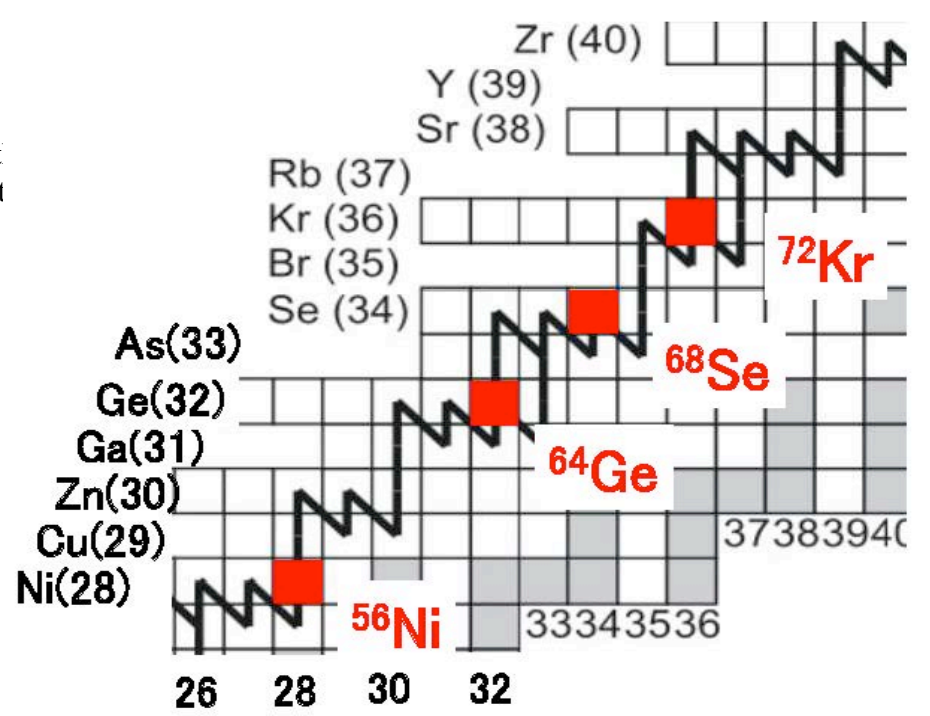


instance at ${ }^{72} \mathrm{Kr}$ in the figure, the beta decay of ${ }^{72} \mathrm{Kr}$ would compete with the $2 \mathrm{p}$ process. If the $2 p$ process is very slow, then the rp-process flow has to wait for the beta decay to ${ }^{72} \mathrm{Br}$. Since the half-life of ${ }^{72} \mathrm{Kr}$ is $17.3 \mathrm{sec}$. and the ordinary type I X-ray burst duration time is 20-30 sec, a large portion of the rp-process flow will stall at this point. To this problem, one needs to know the masses of the surrounding nuclei, and also needs to learn the proton capture process at the bottleneck points ${ }^{73} \mathrm{Kr}$ and ${ }^{74} \mathrm{Rb}$.

\subsection{Mass measurement of ${ }^{65} \mathrm{As}$ for the possible waiting point at ${ }^{64} \mathrm{Ge}$}

As discussed above, the masses of atomic nuclei play a crucial role for determining the basic path around the waiting points. High precision mass measurements have been made extensively for the nuclei along the possible pathway of the rp-process, although there still remain unmeasured nuclei. Such high precision mass measurement can be made using an ion storage ring, a Penning trap and an MR-TOF devices.

The first high precision mass measurement of ${ }^{65} \mathrm{As}$ was made at the CSRe ion storage ring at Lanzhou, China. The revolution time of each ions is described by the following equation [16];

$$
\frac{\Delta T}{T}=\frac{1}{\gamma_{t}^{2}} \frac{\Delta(m / q)}{(m / q)}-\left(1-\frac{\gamma^{2}}{\gamma_{t}^{2}}\right) \frac{\Delta v}{v}
$$

The high precision mass measurement will be made by isochronous mass spectrometry, where the ions stored in a storage ring keep the isochronous condition by setting $\gamma=\gamma_{t}$ with $\gamma_{t}$ the transition energy of the ring. Then, the precise $\mathrm{m} / \mathrm{q}$ value will be extracted using reference particles whose masses are known with high precisions. Here, ${ }^{65}$ As nuclides were produced by the fragmentation of ${ }^{78} \mathrm{Kr}$ at $480 \mathrm{AMeV}$, and injected into the CSRe ring together with other ions.

Figure 2 shows a time spectrum obtained in the measurement of ${ }^{65} \mathrm{As}$, where the revolution time of ${ }^{65} \mathrm{As}$ was measured simultaneously with other ions. Experimental result obtained for the mass excess of ${ }^{65} \mathrm{As}$ is -46937 (85) $\mathrm{keV}$. This gives the proton separation energy of $-90(85) \mathrm{keV}$, indicating the nucleus ${ }^{65} \mathrm{As}$ is slightly unbound for proton. Thus, the $2 \mathrm{p}$ process would be easier to go through from ${ }^{64} \mathrm{Ge}$ than thought before.

Fig. 2. The revolution time spectrum for the ${ }^{65} \mathrm{As}$ meassurement [12].

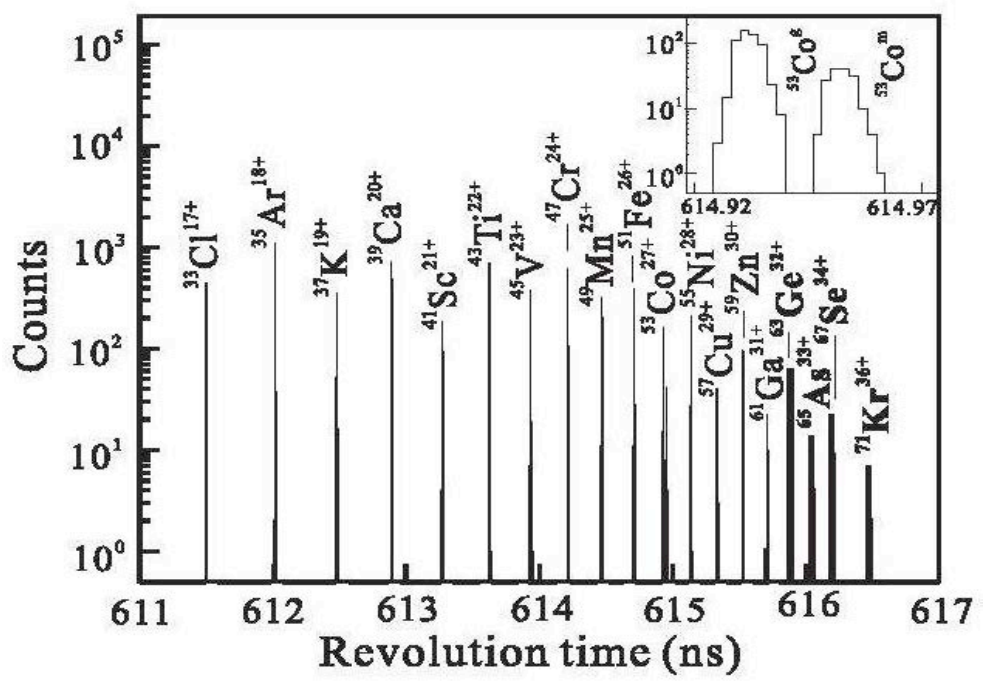




\subsection{Possible waiting point at ${ }^{72} \mathrm{Kr}$}

Recently, an interesting result was obtained for the possible waiting point at ${ }^{72} \mathrm{Kr}$ in the EURICA campaign experiment at RIKEN-RIBF [17]. Figure 1 suggests that ${ }^{72} \mathrm{Kr}$ could be a waiting point nucleus for the rp-process. The rp-process flow would be greatly reduced if the $2 \mathrm{p}$-process from ${ }^{72} \mathrm{Kr}$ is not favoured, as the half-life of ${ }^{72} \mathrm{Kr}$ is comparable to the decay time of the X-ray bursts, as discussed earlier. Figure 3 shows the yield distributions of $\mathrm{N}=35,36$ isotones including ${ }^{73} \mathrm{Rb}$. A smooth distribution was

Fig. 3. The yields of isotones of $\mathrm{N}=35$ and $36.73 \mathrm{Rb}$ was not observed at the experimental target station of EURICA [17].

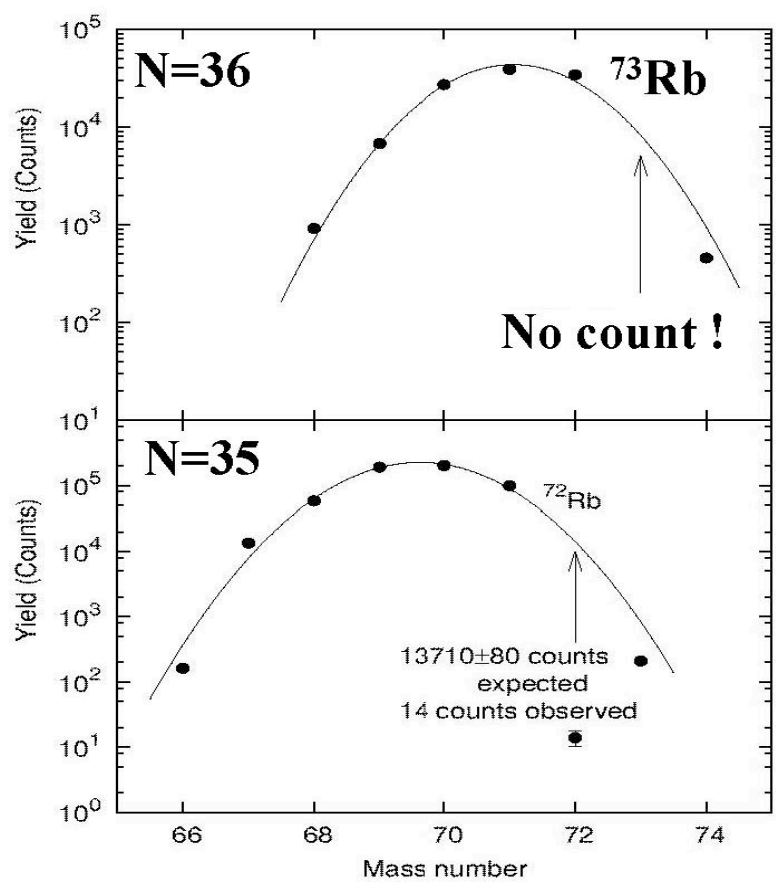

observed except for ${ }^{73} \mathrm{Rb}$ in $\mathrm{N}=36$. Since the measurements were made at the end of the zero-degree spectrometer of RIBF, about $130 \mathrm{~m}$ from the production target, ${ }^{73} \mathrm{Rb}$ is considered to have decayed to ${ }^{72} \mathrm{Kr}$ by a proton emission. The upper limit of the half-life was estimated to be $81 \mathrm{~ns}$. The proton decay probability depends on the decay energy as well as the angular momentum of the shell orbit of the decaying proton in ${ }^{73} \mathrm{Rb}$. Considering these facts together with possible deformation, one can constrain the proton decay energy. Here, the charged particle decay property is defined by the Coulomb penetrability as well as the centrifugal barrier penetrability for low energy proton decay. The estimated proton decay energy is not smaller than about $600 \mathrm{keV}$. Thus, the 2 p-process seems difficult to proceed, suggesting the significant flux might stall at ${ }^{72} \mathrm{Kr}$, although detailed model analysis is needed to conclude on this point.

\section{3 p-Nucleus Production and the Termination Process}


Haettner et al. [13] had reported precision mass measurements of the nuclei near the rp-process path at $A=81-89$, and claimed that the rp-process would terminate at around this mass region, because the $\mathrm{ZrNb}$ cycle would be formed, and thus the rp-process flow would

Fig. 4. The alpha separation energies were calculated based on the new masses determined at Lanzhou. The new data shows a smooth decrease suggesting the $\mathrm{ZrRb}$ cycle[13] would be less effective. [18].

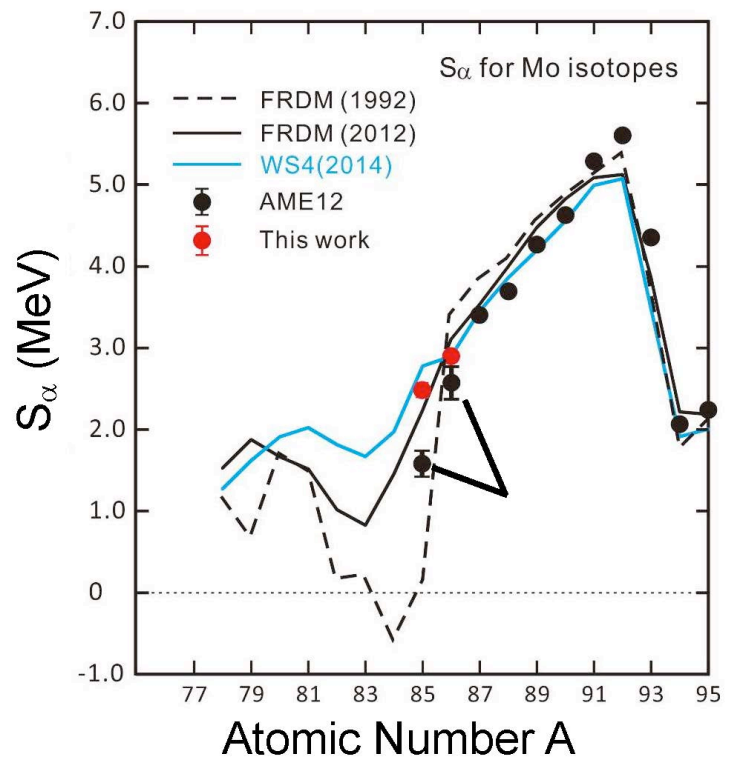

terminate at this point at a certain temperature. However, this conclusion was found to be very uncertain because their analysis included some other masses not measured [13].

Recently, the masses of ${ }^{82} \mathrm{Zr}$ and ${ }^{84} \mathrm{Nb}$ were measured with high precision for the first time, and those of ${ }^{79} \mathrm{Y},{ }^{81} \mathrm{Zr}$, and ${ }^{83} \mathrm{Nb}$ were re-measured at the CSRe storage ring in Lanzhou. Figure 4 shows the alpha separation energy derived based on the new masses, indicating the $\mathrm{ZyNb}$ cycle seems suspicious [18].

These new mass data of Lanzhou have a considerable impact to the p-nuclei production in this mass region, especially for production of ${ }^{84} \mathrm{Sr}$. The vp-process was proposed by three groups [2 - 4], and all of them predict quite a large abundance of ${ }^{84} \mathrm{Sr}$, but now with the new masses including ${ }^{82} \mathrm{Zr}$, the simulation of the $v \mathrm{p}$-process show significantly smaller abundance for ${ }^{84} \mathrm{Sr}$. The new vp-process calculation produces less flow to ${ }^{84} \mathrm{Mo}$, favouring a flow to ${ }^{82} \mathrm{Y}$ at ${ }^{82} \mathrm{Zr}$, resulting in less ${ }^{84} \mathrm{Sr}$ production due to the new masses. Thus, the overproduction of ${ }^{84} \mathrm{Sr}$ was mainly originated due to lack of detailed mass information of the nuclei around $A=84$. This demonstrates that precision mass determination is very crucial for studying the p-nucleus production in the $\mathrm{vp}$-process.

\section{Summary and Scope}

Explosive hydrogen burning process is one of the hottest subjects both for astronomical observation as well as for nuclear physics. Since nuclear reactions runs through proton-rich nuclear region in the explosive hydrogen burning process, this process has a great chance to contribute for p-nucleus production in the medium mass region. The most critical, physical parameters, the masses of nuclei along the possible pathway have been extensively measured, but still are not yet fully measured with precision. In the coming years, not only masses of nuclides, but also the proton capture processes at the bottle necks would become 
the next important subject for experiments to get full nuclear physics information to understand the hydrogen burning condition, and to know the contribution for p-nuclei.

As for the vp-process, one another important subject is to study the neutron induced reactions around the waiting point nuclei [11]. The $(n, p)$ and $(n, \gamma)$ reactions would discard the waiting points and accelerate the burning flow toward heavier mass region. These reactions are totally unknown.

The mechanism of type I X-ray burst will be further investigated not only by nuclear physics experiment, but also by astronomical observation which will tell us directly the heavy element synthesis in the bursts.

\section{References}

1. Proceedings of the $14^{\text {th }}$ International Symposium on Nuclei in the Cosmos NIC2016, JPS Conference Proceedings No. 14 (2017) ed. S. Kubono, T. Kajino, S. Nishimura, T. Isobe, S. Nagataki, T. Shima, and Y. Takeda

2. R. K.Wallace and S. E.Woosley, Astrophys. J. Suppl. Ser. 45, 389 (1981)

3. H. Schatz, et al., Phys. Rev. Lett. 86, 3471 (2001)

4. B.P. Abbott, et al., Phys. Rev. Lett. 119, 161101 (2017)

5. L.-X. Li and B. Paczn/sky, Astrophys. J. Lett. 509, L59 (1998)

6. C. Fröhlich et al., Phys. Rev. Lett. 96, 142502 (2006)

7. S. Wanajo, Astrophys. J. 647, 1323 (2006)

8. J. Pruet et al., Astrophys. J. 244, 1028 (2006)

9. M. Arnould and S. Goriely, Phys. Rep. 384 (2003) 1

10. S. Wanajo, H.-T. Janka, and S. Kubono, Astrophys. J. 729, 46 (2011)

11. S. Kubono. Proc. $8^{\text {th }}$ European Summer School on Experimental Nuclear Astrophysics, IOP Publishing Journal of Physics: Conference Series 703, 012009 (2016)

12. X.L. Tu et al, Phys. Rev. Lett. 106, 112501 (2011)

13. E. Haettner et al., Phys. Rev. Lett. 106, 122501 (2011)

14. R.H. Cyburt, et al., Atrophys. J. 830, 55 (2016)

15. C.E. Rolfs and W.S. Rodney, Cauldrons in the Cosmos, The Univ. Chicago Press, 1988

16. X.L. Tu, et al., Nucl. Instr. Method A 654, 213 (2011)

17. H. Suzuki, Phys. Rev. Lett. 119, 192503 (2017)

18. Y.H. Zhang, et al., Proceedings of the Origin of Matter and Evolution of Galaxies 2017, Daejeon, Korea, 2017, to be published in AIP Conf. series 